PART SECOND.

\title{
abibliographical æRecord.
}

Ant. I.-The Principles and Practice of Operative Surgery. By Frederick C. SKey; F.R.S., Surgeon to St. Bartholomew's Hospital, \&c. \&c. Second Edition.-London, 1858. pp. 732.

FEw English surgeons have ventured to write a systematic work on surgery. However large the experience of any individual may be, it cannot be sufficient to enable him to write originally upon all the subjects which are usually included in a complete treatise on any branch of the healing art. The nearest approaches to good systems of surgery are those works in which the labours of many authors are compiled, but even here, from the limited space allowed to each subject, the meaning of the original author is often obscured, and much that will not bear a critical examination becomes mixed up with that which is true. Mr. Skey's work is evidently the result of his own experience, and as such we must not be disappointed if we find in it the views of other surgeons imperfectly represented. Mr. Skey's work is, in fact, a series of practical dissertations upon those points which, in the practice of a large hospital, have fallen under his own notice. From the merit of these essays we would in no way detract, if we venture to point out instances in which Mr. Skey's experience is at variance with that of other surgeons, or where subjects deemed generally of importance are inadequately described.

After some introductory observations of an ethical character, the author proceeds to discuss the various circumstances and phenomena attending the performance of an operation. He observes that-

"At a period when the functions of the animal economy were less understood, the value of blood was less highly estimated than at present. It has now become a recognised rule in practice, to exercise the utmost caution in preventing unnecessary loss. ... . . Just in proportion to the quantity of blood which escapes from the circulation during an operation on a healthy person, always supposing the quantity to be considerable, may we anticipate difficulties and obstructions in our after-treatment of the case. . . . In children, no precaution tending to the prevention of loss of blood should be neglected. (p. 24.)

The importance of this subject is becoming more and more recognised by English surgeons, and we believe that Mr. Skey has done good service by directing the attention of the profession particularly
to it. 
In the treatment after operations, Mr. Skey dwells upon the propriety of administering nutritious food, and, where the appetite allows of it, he prefers solid food to beef-tea and soups. When thirst is present, slightly nutritious drinks should be given, such as whey, or milk and water. Constipation Mr. Skey regards as a general consequence of loss of blood, and therefore no demand should be made upon the constitution by purgatives, until the deficiency is in some measure supplied.

The subject of dislocations is treated at length, and with much clearness, Mr. Skey here gives the same valuable suggestions as the results of his own extended experience-

"In dislocations of the femur on the dorsum ilii, we do not attempt to draw the bone in a direct line with the acetabulum, but we carry it below round its back and elevated margin, and no sooner does it reach the lower part of the rim, which is much less prominent than the upper and back part, than the muscles immediately restore it to its socket. The same rule holds in dislocation of the ulna and radius backward, at the elbow-joint. I believe the exact line of extension to be much more readily determined, and in truth, a less important subject of consideration, than it is generally deemed. I believe that, if we bring the bone sufficiently downwards, and place it in the neighbowihood of the articulation, the muscles alone will replace it without difficulty." (p. 81.)

With regard to dislocation of the head of the humerus, Mr. Skey observes-

"With the heel in the axilla, we make a simple extension of the arm from the wrist; we leave the scapula unfettered to assume its own direction; and by the extending power slightly, as it is applied by one person only, the scapula is made to revolve on its centre, through the leverage exercised on the acromion by the deltoid. . . . . I have seen the protracted efforts of pulleys, drawing outward, fail again and again with the utmost effort, when the head of the bone has been restored to the glenoid cavity by the single agency of one person subsequently placing his heel in the axilla. There is no reason why, in very muscular subjects or in old dislocations, the same principle may not be applied conjointly with the use of pnlleys. For the purpose of retaining this admirable, because most efficient principle, I employ a well padded iron knob which may represent the heel, from which-there extend laterally two strong branches of the same metal, each ending in a bulb or ring of about four inches in length, the office of which is designed to keep the margins of the axilla as free from pressure as possible. . . . . It is of the utmost importance, if we wish for success, that we have the margins of the axilla untrammelled, in order that the bone (the scapula) be allowed to descend unchecked, at least by our agency." (pp. 106, 10\%.)

Mr. Skey throughout this chapter dwells upon the importance of the principle that the general extension of the muscles in the axis of the affected bone ought to be adopted, for the purpose of bringing a bone downwards, within the range of their general, and not their individual action, and that we gain nothing by the resort to any direct line of traction, by which we give relief to one or more muscles at the expense of others.

Chapter VI. of Mr. Skey's work treats at length on the subject of fractures, and in the course of his observations many excellent remarks occur. Here again, however, the author confines himself to his own experience, and he has failed to avail himself of the improvements 
which have been going on elsewhere. In the section on fractures of the leg, for instance, $\mathrm{Mr}$. Skey gives a woodcut of a swing cradle, which no doubt is a great improvement upon some cradles formerly used. It admits of the leg moving from side to side without any disturbance of the fracture, but it is essentially defective in not allowing the corresponding motions as the patient gets higher or lower in the bed. Mr. Salter's swing, now in general use, contrasts in this respect most favourably with that which is represented in Mr. Skey's work.

In the chapter on Wounded Arteries, Mr. Skey gives a very clear account of the two great theories by which surgeons have usually accounted for the obliteration of an artery, by ligature-

"The principle laid down by Dr. Jones, and deduced from his interesting experiments, is that the union of the opposite sides of an artery is effected by the lymph thrown out from the divided coats, both within the tube of the artery and between the coats themselves, and that this lymph becomes accessory to the consolidation and to the final obliteration of the vessel." (p. 205.)

Scarpa, on the other hand, asserts that apposition alone will ensure perfect adhesion of the opposite surfaces-

"That the artery does not become obliterated by the organization of lymph, thrown out by the divided tissues along the circle of the ligature, and in its immediate neighbourhood, by a process of adhesive inflammation, uniting the opposing surfaces of the serous coat."

Mr. Skey does not give his own opinion upon the subject, nor does he intimate to which of the above theories he inclines; we believe neither of them to be strictly correct. It has of late years been demonstrated fully, that the lining membrane of an artery or of a vein will not, as long as it maintains its integrity, be made to secrete lymph by any process of mechanical irritation; but we all know how readily the fibrin of the blood is deposited upon any foreign substance, or upon any irregular or abraded surface within the vascular system. All analogy would thus lead us, even were direct experiment wanting, to say, that the first bond of union in a ligatured artery is the fibrin deposited from its contained blood, and not the lymph effused from its inflamed coats. With regard to the second supposition, that of the adhesion of the opposed surfaces of the serous coats, it might be sufficient to remark that in no part of the body is the simple apposition of two serous surfaces ever followed by union without some uniting medium ; but if direct experiment were wanting, we have it supplied in the length of time that both arteries and veins will remain with their cavities closed by external pressure without there being the least indication of the adhesion of their opposed surfaces.

The principle of the obliteration of an artery by the fibrin deposited from its contained blood, is allowed by everybody, including $\mathrm{Mr}$. Skey, in other cases, as for instance in aneurism. Why not, then, allow the principle of union by fibrin, as well where the internal coat of an artery has been divided by ligature, as where it has been destroyed by disease?

"The process of union of the opposite sides of an artery and its consequent obliteration is, however, not to be determined by the period of the separation 
of the ligature, which may be protracted by other causes. . . . . In his experiments upon this subject, Mr. Travers has proved the removal of a ligature, within even a few hours of the operation for applying it, may be effected with safety, and the entire obliteration of the vessel secured. But as a general rule, it is better, because safer, to leave the ligature to come away by itself." (p. 237.)

With this opinion we cordially agree. One remarkable case has been published in which, after a temporary ligature applied in the way above mentioned, gangrene of the foot speedily followed its removal. This curious phenomenon can probably only be accounted for on the supposition that the period during which the artery was compressed was not sufficient to allow the coagula fully to form, and that when the current was re-established, the semi-coagulated blood was carried into the distal arteries and stagnated in their capillaries. It is not unimportant also to notice in connexion with this subject, that in several cases where compression for the cure of aneurism has been used, and subsequently relaxed, mortification, commencing in the distal extremity of the limb, has followed. These have been, however, altogether exceptional cases, and in no way militate against the value of compression as an ordinary mode of treating aneurism.

For the purpose of obliterating varicose veins, Mr. Skey still adheres to the old plan of destroying a portion of the skin over the vein with caustic. It is painful and tedious, and is now very generally supplanted by the simpler plan of the prolonged compression of the vein, or the still quicker plan of subcutaneous section.

Chapter XII. treats of amputations. Three forms of amputation are here described-the circular, the flap, and that which is commenced by an incision through the integuments, only forming a double flap of skin. Mr. Teale's operation is not included. Most of the observations which we should have to make on this head have already been anticipated in a review of Mr. Teale's work in a former number. We are still inclined to regard Mr. Teale's mode of amputating as superior to any of those described by Mr. Skey, but experience has proved that even this is not free (as we were at first led to suppose) from the common consequences of amputation, namely, phlebitis, secondary deposits, erysipelas, \&c. While alluding to this subject, we may remark how very scanty and imperfect the descriptions of these diseases are in Mr. Skey's work. In fact, Mr. Skey professes not to have seen a case of pyæmia in his wards for the last three years. This assertion contrasts strangely with the experience of other surgeons. In a little work recently published by Mr. A. G. Sansom, "tables of mortality are given, derived from various sources, showing the percentage of those who die after different operations. In one of these tables, No. VI., no less than one-fourth of the cases of primary amputation of the leg are reported to have died from pyæmia alone. We are, then, at a loss to account for the fact of Mr. Skey's not having witnessed this disease for so long a period.

We are unable to follow Mr. Skey through the other chapters of his

* The Mortality after Operations of Amputation of the Extremities. By A. E. Sansom. Prize Essay. London, 1859. 
work, every part of which contains original observations and interesting remarks. Although the work does not, as a whole, represent English surgery of the present day, we are of opinion that the independence of thought and research which characterize it, give it a peculiar merit, and justify our recommending it to every earnest student of his profession.

Anт. II.-Selections from favourite Prescriptions of Living American Practitioners. By Horace Green, M.D., LL.D., President of the Faculty, and Emeritus Professor of the Theory and Practice of Medicine in the New York Medical College, \&c.-New York, 1858. pp. 206.

OuR readers are familiar with the name of Dr. Horace Green as the great promoter of the local treatment of morbid conditions of the fauces and air-passages. In the above work he comes before us in a new capacity, and offers to present us with a picture of American practice, so far as it is capable of being illustrated by prescriptions, and the remarks that they call for. The contents of the work scarcely correspond to the title; the latter leads the reader to expect that the bulk of the prescriptions at least will be by other American practitioners than the author, whereas they form but a small portion of the volume, Dr. Green's own formulæ being those that are put prominently forward; while in the majority of cases the prescriptions of others are quoted as being " highly recommended by an intelligent and experienced practitioner" - as being employed by "a physician of great experience"-by an "eminent physician of New Orleans"-by a "physician of this State of large experience," and the like impersonal individualities which excite no interest and leave no impression. Of the first hundred formulæ, sixty-five appear to have emanated from the pen of Dr. Green, while to eight only of the remaining thirty-five the authors' names are appended. The prescriptions are classified according to their assumed therapentic effect, and are distributed in fourteen chapters, as narcotics, tonics, excitants, cathartics, emetics and expectorants, astringents, diaphoretics, diuretics, antispasmodics, anthelmintics, emmenagogues, gargles and lotions, antacids and antilithics, and derivatives. The majority of the prescriptions are closely analogous to what we habitually see in this country; more so than what those might have anticipated who are acquainted with the universal Formulary by Doctors Griffith and Thomas.* There are, however, two drugs which the anthor specially recommends, and regarding which we would therefore say a word or two, as they are not in common use in this country. One is the root of the Podophyllum peltatum, or May apple, which is regarded as a vegetable mercurial in its operation; it is prescribed in the form of an extract, or the active principle is isolated as podophyllin. ${ }^{\dagger}$ The former is administered in grain doses; of the latter half a grain is given at a time. We subjoin two of the formulæ:

* See British and Foreign Medico-Chirurgical Review, Oct. 1855, p. 462.

$\dagger$ The Record of Pharmacy and Therapeutics, edited by Dr. Medlock, informs us that Podophyllin may be obtained at the General Apothecaries' Company, in Berners-street. 